\title{
Generation of Narrow-Band Hyperentangled Nondegenerate Paired Photons
}

\author{
Hui Yan, Shanchao Zhang, J.F. Chen, M. M. T. Loy, G. K. L. Wong, and Shengwang Du* \\ Department of Physics, The Hong Kong University of Science and Technology, Clear Water Bay, Kowloon, Hong Kong, China
}

(Received 4 November 2010; published 21 January 2011)

\begin{abstract}
We report the generation of nondegenerate narrow-bandwidth paired photons with time-frequency and polarization entanglements from laser cooled atoms. We observe the two-photon interference caused by Rabi splitting with a coherence time of about $30 \mathrm{~ns}$ and a visibility of $81.8 \%$ which verifies the timefrequency entanglement of the paired photons. The polarization entanglement is confirmed by polarization correlation measurements which exhibit a visibility of $89.5 \%$ and characterized by quantum-state tomography with a fidelity of $90.8 \%$. Taking into account the transmission losses and duty cycle, we estimate that the system generates hyperentangled paired photons into opposing single-mode fibers at a rate of 320 pairs per second.
\end{abstract}

DOI: 10.1103/PhysRevLett.106.033601

Entangled paired photons, a hallmark tool of quantum optics, play a critical role in quantum information processing, quantum computation and quantum communication [1]. For many decades, spontaneous parametric downconversion (SPDC) in nonlinear crystals has been used as the standard method to generate nonclassical correlated photons [2,3]. Recently, much research interest has been drawn to the production of photons that are simultaneously entangled in more than one degree of freedom, or hyperentangled [4-8], because of the increasing information carrying capacity of these paired photons. The hyperentangled photons can be implemented as building blocks for efficient quantum computation $[9,10]$. However, the SPDC sources typically have wide bandwidths $(\sim \mathrm{THz})$ and short coherence time $(\sim$ ps $)$, and thus are not suitable for use in entanglement storage based long distance quantum communication protocols. By putting the nonlinear crystal inside a cavity and using two-photon interference outside the cavity, generation of narrow-band degenerate polarizationentangled photon pairs has been demonstrated [11].

Following the protocol of long distance quantum communication proposed by Duan et al. [12], nonclassical photon pairs have been generated from atomic ensembles using "writing-reading" technique [13,14]. Polarization entanglement has been achieved using this technique [15], but the photons are not entangled in time-frequency due to the fact that "writing" and "reading" are timeseparated pulsed processes. Subsequently, Harris group [16-18] generated narrow-bandwidth time-frequency entangled paired photons with the two driving lasers running in continuous-wave (cw) mode, making use of electromagnetically induced transparency [19] and spontaneous four-wave mixing (SFWM). With the long coherence time of these biphotons, it was possible to shape and modulate their single- and two-photon waveforms $[20,21]$. So far, paired photons with entanglement in both time-frequency and polarization have not been produced from atomic
PACS numbers: 42.50.Dv, 03.67.Bg, 03.67.Mn, 42.65.Lm

ensembles, even though this possibility has been suggested theoretically [22].

In this Letter, we report the experimental demonstration of generation of narrow-bandwidth nondegenerate paired photons with both time-frequency and polarization entanglements from a cold atomic ensemble via SFWM. These hyperentangled photons having tunable optical frequencies near atomic resonances, are ideal for performing interaction with and storage using atomic quantum memories [12], and may find application in dense coding quantum communication and computation systems $[9,23]$.

The hyperentangled biphotons are generated from cold ${ }^{85} \mathrm{Rb}$ atoms in a two-dimensional (2D) magneto-optical trap (MOT) with a longitudinal length $L=1.5 \mathrm{~cm}$ and a temperature of about $100 \mu \mathrm{K}$. Figure 1 shows the relevant ${ }^{85} \mathrm{Rb}$ D2-line $(780 \mathrm{~nm})$ energy level diagram and the experimental configuration. The atoms are prepared in the ground level $|1\rangle$. A strong pump laser $\left(\omega_{p}\right)$, with an
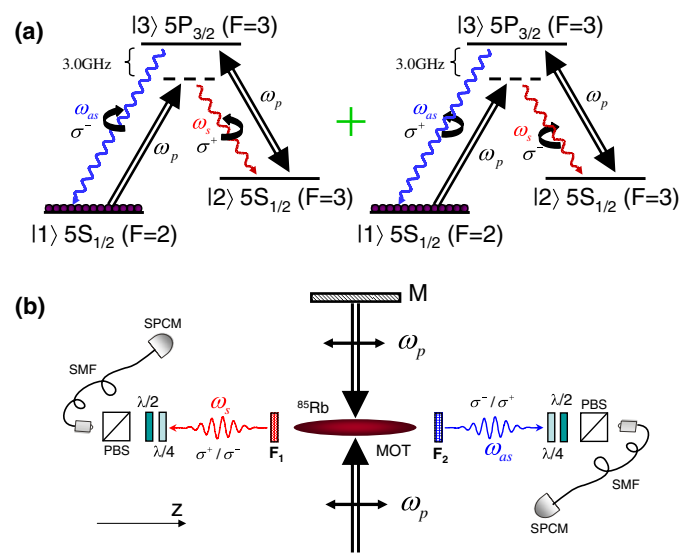

FIG. 1 (color online). Schematics of narrow-band nondegenerate hyperentangled paired photon generation. (a) ${ }^{85} \mathrm{Rb}$ energy level diagram with two possible polarization configurations for the spontaneously emitted photon pairs. (b) Experimental setup with a right-angle geometry. 
elliptical beam profile $\left(1 / e^{2}\right.$ major and minor diameters are 13.0 and $0.8 \mathrm{~mm}$ ) that matches the size of atom cloud, is sent through the 2D MOT transversely and then retroreflected back by a mirror $(M)$. The pump laser beam is linearly polarized parallel to the longitudinal symmetry $z$ axis of the MOT and red-detuned from the transition $|1\rangle \rightarrow$ $|3\rangle$ by $3 \mathrm{GHz}$ so that it coincides with the resonance transition $|2\rangle \leftrightarrow|3\rangle$. The counterpropagating phasematched paired Stokes $\left(\omega_{s}\right)$ and anti-Stokes $\left(\omega_{\text {as }}\right)$ photons are emitted along the $z$ axis and at $90^{\circ}$ to the pump beams. They are collected by two opposing single-mode fibers (SMF) and detected by the single-photon counter modules (SPCM, Perkin Elmer SPCM-AQ4C). The two-photon correlation is analyzed using a time-to-digital converter (Fast Comtec P7888). The Stokes and anti-Stokes single modes are focused at the MOT center with a $1 / e^{2}$ diameter of $0.22 \mathrm{~mm}$. Here, the pump laser acts not only as the pump beams of the SFWM process but also as the coupling laser for creating EIT for the anti-Stokes photons. Because of the hyperfine splitting $\Delta_{12}=2 \pi \times 3 \mathrm{GHz}$ between $|1\rangle$ and $|2\rangle$, the Stokes and anti-Stokes photons are nondegenerate and their central optical frequencies differ by $6 \mathrm{GHz}$, i.e., $\omega_{\text {as } 0}-\omega_{s 0}=2 \Delta_{12}$. Two narrow-band optical frequency filters, $F_{1}$ and $F_{2}(0.5 \mathrm{GHz}$ bandwidth $)$, with peak transmissions centered at $\omega_{s 0}$ and $\omega_{\text {as } 0}$ respectively are used to distinguish the Stokes and anti-Stokes photons. Two pairs of quarter-wave $(\lambda / 4)$ and half-wave $(\lambda / 2)$ plates are placed in opposing beam paths for measuring polarization correlation and quantum-state tomography.

The time-frequency entanglement between the Stokes and anti-Stokes photons is a result of energy conservation: $\omega_{s}+\omega_{\text {as }}=2 \omega_{p}$ when the atoms are driven by counterpropagating $\mathrm{cw}$ pump beams, which allow spontaneously generated paired photons to emit at right-angle under perfect phase-matching condition. To produce entanglement in polarization, we make use of the degenerate Zeeman sub states of each hyperfine energy level. By choosing the 2D MOT longitudinal symmetry axis as the quantization $z$ axis, Zeeman states with $\Delta M_{F}=0$ are coupled by the linearly polarized pump beams. Conservation of angular moment along the $z$ axis allows two possible circular polarization configurations as shown in Fig. 1(a): $\left|\sigma_{s}^{+} \sigma_{\text {as }}^{-}\right\rangle$and $\left|\sigma_{s}^{-} \sigma_{\text {as }}^{+}\right\rangle$. The hyperentangled quantum-state of the paired Stokes and anti-Stokes photons at the two detectors are described by

$$
\begin{aligned}
\left|\Psi_{s, \mathrm{as}}\left(t_{s}, t_{\mathrm{as}}\right)\right\rangle= & \psi(\tau) e^{-i \omega_{\mathrm{s} 0} t_{s}} e^{-i \omega_{\mathrm{as} 0} t_{\mathrm{as}}} \frac{1}{\sqrt{2}}\left(\left|\sigma_{s}^{+} \sigma_{\mathrm{as}}^{-}\right\rangle\right. \\
& \left.+\left|\sigma_{s}^{-} \sigma_{\mathrm{as}}^{+}\right\rangle\right),
\end{aligned}
$$

where $\tau=t_{\mathrm{as}}-t_{s}$ is the relative time delay between the paired photons.

We conduct the experiment periodically with a MOT time of $4.5 \mathrm{~ms}$ and a biphoton generation window of $0.5 \mathrm{~ms}$ at each period. Near the end of each trapping cycle, we optically pump all atoms to the ground level $|1\rangle$. The 2D quadruple magnetic field, with zero field along the longitudinal axis, has a transverse gradient of $10 \mathrm{G} / \mathrm{cm}$. This allows us to keep the quadruple magnetic field on continuously during the experiment. Our system is significantly simpler than other atom-trap systems where the magnetic field must be switched on and off frequently [15] to ensure polarization entanglement.

We first verify the time-energy entanglement of the paired photons as follows. We measure the time correlation function of Stokes and anti-Stokes photons at their circular polarizations of $\sigma_{s}^{+}$(or $\sigma_{s}^{-}$) and $\sigma_{\text {as }}^{-}$(or $\sigma_{\text {as }}^{+}$). We take a dressed-state picture to explain the physics involved. With the presence of the on-resonance coupling (pump) laser, the ground levels $|2\rangle$ and $|3\rangle$ are split into two with a frequency separation equal to the coupling laser Rabi frequency $\Omega_{c}$. This results in two SFWM channels as shown in Fig. 2(a). In the case of strong coupling laser power and low optical depth, the single-photon spectrum can be calculated according to FWM nonlinear interaction [24] and plotted in Fig. 2(b), which displays two resonance peaks separated by $\Omega_{c}$ along the angular frequency axis. The widths of these two resonance peaks are determined by $2 \gamma_{13}=2 \pi \times 6 \mathrm{MHz}$ where $\gamma_{13}$ is the electric dipole relaxation rate between $|1\rangle$ and $|3\rangle$. The generated Stokes and anti-Stokes photon pair can be described as the entangled state $\left(\left|\omega_{s 0}+\Omega_{c} / 2\right\rangle\left|\omega_{\text {as } 0}-\Omega_{c} / 2\right\rangle-\left|\omega_{s 0}-\Omega_{c} / 2\right\rangle \mid \omega_{\text {as } 0}+\right.$ $\left.\left.\Omega_{c} / 2\right\rangle\right) / \sqrt{2}$. Following the theoretical procedure given in Ref. [24] for the Rabi oscillation regime, the interference between the two SFWM channels leads to the following two-photon wave packet:

$$
\psi(\tau)=B L \sin \left(\frac{\Omega_{c} \tau}{2}\right) e^{-\gamma_{13} \tau} \Theta(\tau),
$$

where we have taken into account the linewidths of the two resonances. $B$ is a grouped nonlinear coupling coefficient
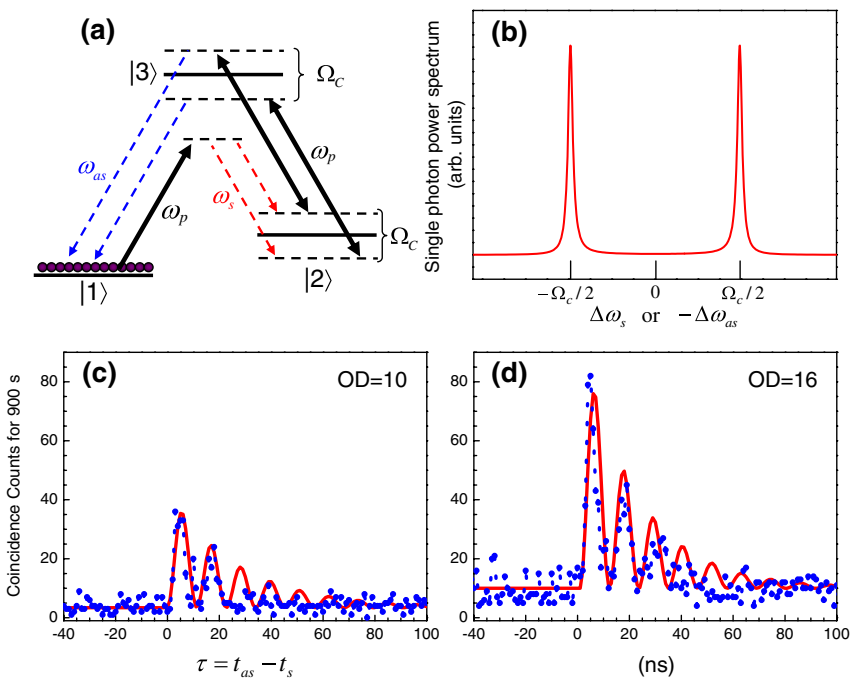

FIG. 2 (color online). (a) The dressed-state picture: two SFWM channels in generating time-energy entanglement. (b) The calculated single-photon spectrum of the Stokes and anti-Stokes photons. (c) and (d) are coincidence counts as a function of the relative time delay at optical depth of 10 and 16 , respectively. 
and $\Theta(\tau)$ is the Heaviside function. The resulting twophoton Glauber correlation function becomes

$$
\begin{aligned}
G_{s, \text { as }}^{(2)}(\tau) & =\left\langle\hat{a}_{s}^{\dagger}(t) \hat{a}_{\mathrm{as}}^{\dagger}(t+\tau) \hat{a}_{\mathrm{as}}(t+\tau) \hat{a}_{s}(t)\right\rangle \\
& =\frac{1}{2}|B L|^{2}\left[1-\cos \left(\Omega_{c} \tau\right)\right] e^{-2 \gamma_{13} \tau} \Theta(\tau),
\end{aligned}
$$

where $\hat{a}^{\dagger}$ and $\hat{a}$ are the photon creation and annihilation operators. Figures 2(c) and 2(d) show the measured twophoton correlation at optical depth (OD) of 10 and 16, respectively. The pump laser has a power of $100 \mathrm{~mW}$ and gives an effective coupling Rabi frequency $\Omega_{c}=2 \pi \times$ $86 \mathrm{MHz}$. The data (blue circular dots) contained in each plot were collected in a counting period of $900 \mathrm{~s}$ with a time bin width of $1 \mathrm{~ns}$. The solid (red) theoretical curves are calculated from Eq. (3) without any free fitting parameters other than the vertical common scaling factor. The agreement between theory and experiment is good and verifies the time-frequency entanglement indicated in Eq. (2). With the maximum coincidence counts $C_{\max }$ and minimum coincidence counts $C_{\min }$ in the correlation function, we can determine the visibility $V=\left(C_{\max }-\right.$ $\left.C_{\min }\right) /\left(C_{\max }+C_{\min }\right)$ of the two-photon interference. We obtain $V=81.8 \%$ and $V=80.0 \%$ for Figs. 2(c) and 2(d), respectively, which are far beyond the requirement for violation of Bell inequality [25].

The quantum nature of the correlation between two single photons can be measured by the violation of Cauchy-Schwartz inequality $[16,26,27]$. In our experiment, the background nonzero floor in Fig. 2(c) or 2(d) is a result of accidental coincidence between uncorrelated Stokes and anti-Stokes photons from different pairs. The two-photon coincidence rate can be expressed as $R_{s, \text { as }}(\tau)=R_{s} R_{\text {as }}+G_{s, \text { as }}^{(2)}(\tau)$, where $R_{s}$ and $R_{\text {as }}$ are the single-photon rates. We then determine the normalized cross-correlation function $g_{s, \text { as }}^{(2)}(\tau)=R_{s, \text { as }}(\tau) /\left(R_{s} R_{\text {as }}\right)=$ $1+G_{s, \text { as }}^{(2)}(\tau) /\left(R_{s} R_{\mathrm{as}}\right)$. The normalized autocorrelations are given similarly as $g_{s, s}^{(2)}(\tau)=1+G_{s, s}^{(2)}(\tau) / R_{s}^{2}$ and $g_{\mathrm{as}, \mathrm{as}}^{(2)}(\tau)=1+G_{\mathrm{as}, \mathrm{as}}^{(2)}(\tau) / R_{\mathrm{as}}^{2}$. A classical source is bounded by the Cauchy-Schwartz inequality $\left[g_{s, \text { as }}^{(2)}(\tau)\right]^{2} /$ $\left[g_{s, s}^{(2)}(0) g_{\text {as,as }}^{(2)}(0)\right] \leq 1$ [26]. From Fig. 2(c) and 2(d), we get $g_{s, \text { as }}^{(2)}(\tau)$ that peaks at 10 and 9 , respectively. With the measured autocorrelations $g_{s, s}^{(2)}(0) \simeq g_{\mathrm{as}, \mathrm{as}}^{(2)}(0) \simeq 1.0$ using a fiber beam splitter, we obtain a violation of the inequality by a factor of 100 and 81 , respectively.

We next verify the polarization entanglement. To do so, we first convert the circular polarization basis $\left|\sigma_{s}^{+} \sigma_{\text {as }}^{-}\right\rangle$,

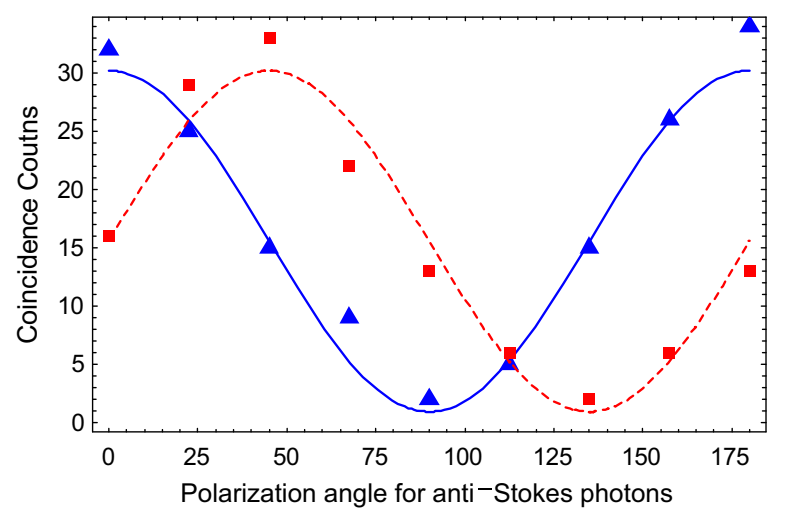

FIG. 3 (color online). Polarization correlation of the paired Stokes and anti-Stokes photons. The polarization angle for Stokes photons are fixed at $0^{\circ}$ and $-45^{\circ}$ for the triangle $(\triangle$, blue) and square $(\square$, red) experimental data, respectively. The solid and dashed are theoretical fitted curves.

$\left|\sigma_{s}^{-} \sigma_{\mathrm{as}}^{+}\right\rangle,\left|\sigma_{s}^{+} \sigma_{\mathrm{as}}^{+}\right\rangle$and $\left|\sigma_{s}^{-} \sigma_{\mathrm{as}}^{-}\right\rangle$into linear polarizations $|H H\rangle,|V V\rangle,|H V\rangle$ and $|V H\rangle$ respectively by passing the photons through the quarter-wave plates as shown in Fig. 1(b). Then we use a half-wave plate followed by a cubic polarization beam splitter (PBS) as the polarization selector. The measured two-photon polarization correlations are displayed in Fig. 3, where the triangle data ( $\triangle$, blue) points are measured by fixing the Stokes photon polarization angle (after the half-wave plate) at $0^{\circ}$ and the square data $\left(\square\right.$, red) at $-45^{\circ}$. Each data point corresponds to the counts collected in $900 \mathrm{~s}$. The system parameters during measurement remain the same as those shown in Fig. 2(c). The solid cosine- and dashed sine-wave curves are theoretical fits with background and amplitude being adjustable parameters. We obtain a visibility $V=89.5 \%$ which violates the Bell-CHSH inequality and indicates the expected maximum polarization entanglement [28].

In order to obtain a complete characterization of the polarization entanglement, we also make a quantum-state tomography to determine the density matrix of the biphoton polarization state $[29,30]$. We add an additional quarter-wave plate between the first quarter-wave plate and the half-wave plate in each beam path. In this way, we can access all 16 independent projection states by simply adjusting this quarter-wave plate and the halfwave plate in both paths. The density matrix is constructed from these 16 independent coincidence counts using the maximum likelihood estimation method [30]:

$$
\left(\begin{array}{cccc}
0.03 & 0.04 & 0.06+0.01 i & -0.01 i \\
0.04 & 0.47 & 0.44-0.02 i & -0.05-0.02 i \\
0.06-0.01 i & 0.44+0.02 i & 0.47 & -0.03-0.01 i \\
0.01 i & -0.05+0.02 i & -0.03+0.01 i & 0.03
\end{array}\right)
$$

whose graphical representation is shown in Fig. 4. The result shows a fidelity of $90.8 \%$ between our measured state and the ideal EPR-Bell state $\frac{1}{\sqrt{2}}\left(\left|\sigma_{s}^{+} \sigma_{\text {as }}^{-}\right\rangle+\right.$ $\left.\left|\sigma_{s}^{-} \sigma_{\text {as }}^{+}\right\rangle\right)$. We also use the density matrix to test violation 

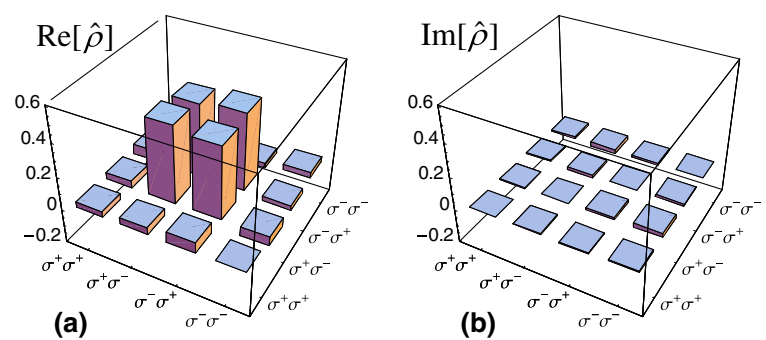

FIG. 4 (color online). Tomography measurement result of the polarization-entangled biphotons: (a) Real and (b) imaginary parts of the density matrix.

of the Bell-CHSH inequality $(|S|<2)$ and obtain $S=2.47 \pm 0.15$.

We notice that, from the symmetry of the right-angle configuration in Fig. 1(b), the counter-propagating Stokes and anti-Stokes photons can go to both directions if the filters are removed. We verify this by exchanging the positions of filters $F_{1}$ and $F_{2}$ and obtain the same results as shown in Figs. 2-4. As discussed previously, we are working in the Rabi oscillation regime [24], where the biphoton emission rate is proportional to square of the atomic optical depth, as shown in Figs. 2(c) and 2(d). Taking into account of the detector quantum efficiency of $50 \%$, fiber-fiber coupling efficiency of $70 \%$, filter transmission of $70 \%$ and duty cycle of $10 \%$, as well as tracing over the time, polarization and momentum spaces, we estimate that our system spontaneously generate 320 pairs per second in the case of Fig. 2(d). The two-photon coherence time is about $30 \mathrm{~ns}$, which corresponds to a bandwidth of about $6 \mathrm{MHz}$.

In summary, we have demonstrated the generation of narrow-bandwidth nondegenerate hyperentangled paired photons with entanglement in both time frequency and polarization from a 2D MOT. We observe a damped Rabi oscillation (with a visibility of $81.8 \%$ ) in the two-photon correlation function that results from the entanglement of two narrow-band $(6 \mathrm{MHz})$ frequency lines. The polarization entanglement is verified by the polarization correlation measurement which exhibits a visibility of $89.5 \%$. We also perform a quantum-state tomography measurement to determine the density matrix and obtain a fidelity of $90.8 \%$. In contrast to the previous experiment [11] where the polarization entanglement is produced from two-photon interference involving passive beam splitters, the timefrequency and polarization entanglements in our work are generated from the internal symmetries of the atomic system. For the first time we show that polarization entanglement can be preserved maximally without switching off the quadruple magnetic field in the 2D MOT configuration. These narrow-bandwidth hyperentangled photons having a coherence time of about $30 \mathrm{~ns}$ are suitable for quantummemory-based long distance quantum communication.

The work was supported by the Hong Kong Research Grants Council (Project No. HKUST600809, No. DAG08/ 09.SC02, and No. DAGS09/10.SC06). Some equipment used in the experiment were provided by the Joyce M. Kuok Lasers and Photonics Laboratory and the William Mong Clusters Laboratory.

*dusw@ust.hk

[1] D. Bouwmeester, A. Ekert, and A. Zeilinger, The Physics of Quantum Information (Springer-Verlag, Berlin, 2000).

[2] D. Burnham and D. Weinberg, Phys. Rev. Lett. 25, 84 (1970).

[3] P. G. Kwiat et al., Phys. Rev. Lett. 75, 4337 (1995).

[4] P. G. Kwiat, J. Mod. Opt. 44, 2173 (1997).

[5] J. T. Barreiro et al., Phys. Rev. Lett. 95, 260501 (2005).

[6] J. Chen et al., Phys. Rev. A 77, 053812 (2008).

[7] G. Vallone et al., Phys. Rev. A 79, 030301(R) (2009).

[8] R. Ceccarelli et al., Phys. Rev. Lett. 103, 160401 (2009).

[9] K. Chen et al., Phys. Rev. Lett. 99, 120503 (2007).

[10] W. -B Gao et al., Phys. Rev. Lett. 104, 020501 (2010).

[11] X. H. Bao et al., Phys. Rev. Lett. 101, 190501 (2008).

[12] L. M. Duan et al., Nature (London) 414, 413 (2001).

[13] C. H. van der Wal et al., Science 301, 196 (2003).

[14] A. Kuzmich et al., Nature (London) 423, 731 (2003).

[15] D. Matsukevich and A. Kuzmich, Science 306, 663 (2004).

[16] V. Balić et al., Phys. Rev. Lett. 94, 183601 (2005).

[17] P. Kolchin et al., Phys. Rev. Lett. 97, 113602 (2006).

[18] S. Du et al., Phys. Rev. Lett. 100, 183603 (2008).

[19] S. E. Harris, Phys. Today 50, No. 7, 36 (1997).

[20] P. Kolchin et al., Phys. Rev. Lett. 101, 103601 (2008).

[21] J. F. Chen et al., Phys. Rev. Lett. 104, 183604 (2010).

[22] S. Du et al., Phys. Rev. A 76, 013803 (2007).

[23] C. Wang et al., Phys. Rev. A 71, 044305 (2005).

[24] S. Du, J. M. Wen, and M. H. Rubin, J. Opt. Soc. Am. B 25, C98 (2008).

[25] J. D. Franson, Phys. Rev. Lett. 62, 2205 (1989).

[26] J. F. Clauser, Phys. Rev. D 9, 853 (1974).

[27] J. K. Thompson et al., Science 313, 74 (2006).

[28] J. F. Clauser et al., Phys. Rev. Lett. 23, 880 (1969).

[29] A. G. White et al., Phys. Rev. Lett. 83, 3103 (1999).

[30] D. F. V. James et al., Phys. Rev. A 64, 052312 (2001). 